\title{
ORAL MUCOSITIS IN HEMATOPOIETIC STEM CELL TRANSPLANTATION: INCIDENCE AND PATIENT COMPLIANCE WITH THE PREVENTION
}

\section{Midori Nakagaki ${ }^{1}$, Glen A Kennedy ${ }^{2}$}

1. Pharmacy Department, Royal Brisbane and Women's Hospital, Brisbane, Australia

2. Hematology Department, Cancer Care Services, Royal Brisbane and Women's Hospital, Brisbane, Australia

\section{BACKGROUND}

Oral mucositis $(\mathrm{OM})$ is a common side effect of hematopoietic stem cell transplantation (HSCT).. Oral care, cryotherapy with melphalan and low dose lazer are the only recommended prevention strategies by MASCC guidelines

\section{AIMS}

This study aimed to determine the incidence and severity of OM in patients who underwent different types of HSCT in a single Australian centre.

\section{METHODS}

Patients who underwent HSCT were consecutively included. Daily OM grade was recorded by the nurses using WHO grading of OM. Patients were asked to retrospectively grade their OM severity on their hospital discharges (nil, mild, moderate or severe). They were also asked whether they had used cryotherapy. In addition, they were asked how frequently they used mouthwashes. Prior to HSCT, all patients received OM education and encouraged to use mouthwashes. They were routinely offered cryotherapy if they received melphalan. Sodium bicarbonate (Sodibic) and normal saline (NS) mouthwashes were provided and prescribed four times a day

\section{RESULTS}

\section{Table 1. Patients and regimens}

Total patient number was 57 (auto 10, allo 47)

\begin{tabular}{l|l}
\hline Total $\mathrm{N}=57$ & Auto $\mathrm{N}=10$ \\
\hline BEAM auto $(\mathrm{N}=2)$ & \\
\hline HDM auto $(\mathrm{N}=8)$ & Allo $\mathrm{N}=47$ \\
\hline FluMel allo $(\mathrm{N}=24)$ & \\
\hline FluMel TCZ allo $(\mathrm{N}=3)$ & \\
\hline FluTBI allo $(\mathrm{N}=1)$ & \\
\hline CyTBI allo $(\mathrm{N}=10)$ & \\
\hline FluCy allo $(\mathrm{N}=4)$ & \\
\hline Mel Flu TBI Haplo $(\mathrm{N}=3)$ & \\
\hline FluCy TBI Haplo $(\mathrm{N}=2)$ &
\end{tabular}

\section{CONCLUSIONS}

Despite high compliance for oral care and cryotherapy, oveall $40 \%$ of the patients experienced moderate to severe OM. The incidence was higher in allogeneic patients vs autologous. Myeloabrative CyTBI regimen had the highest incidence. Further study is warranted to improve oral OM prevention.

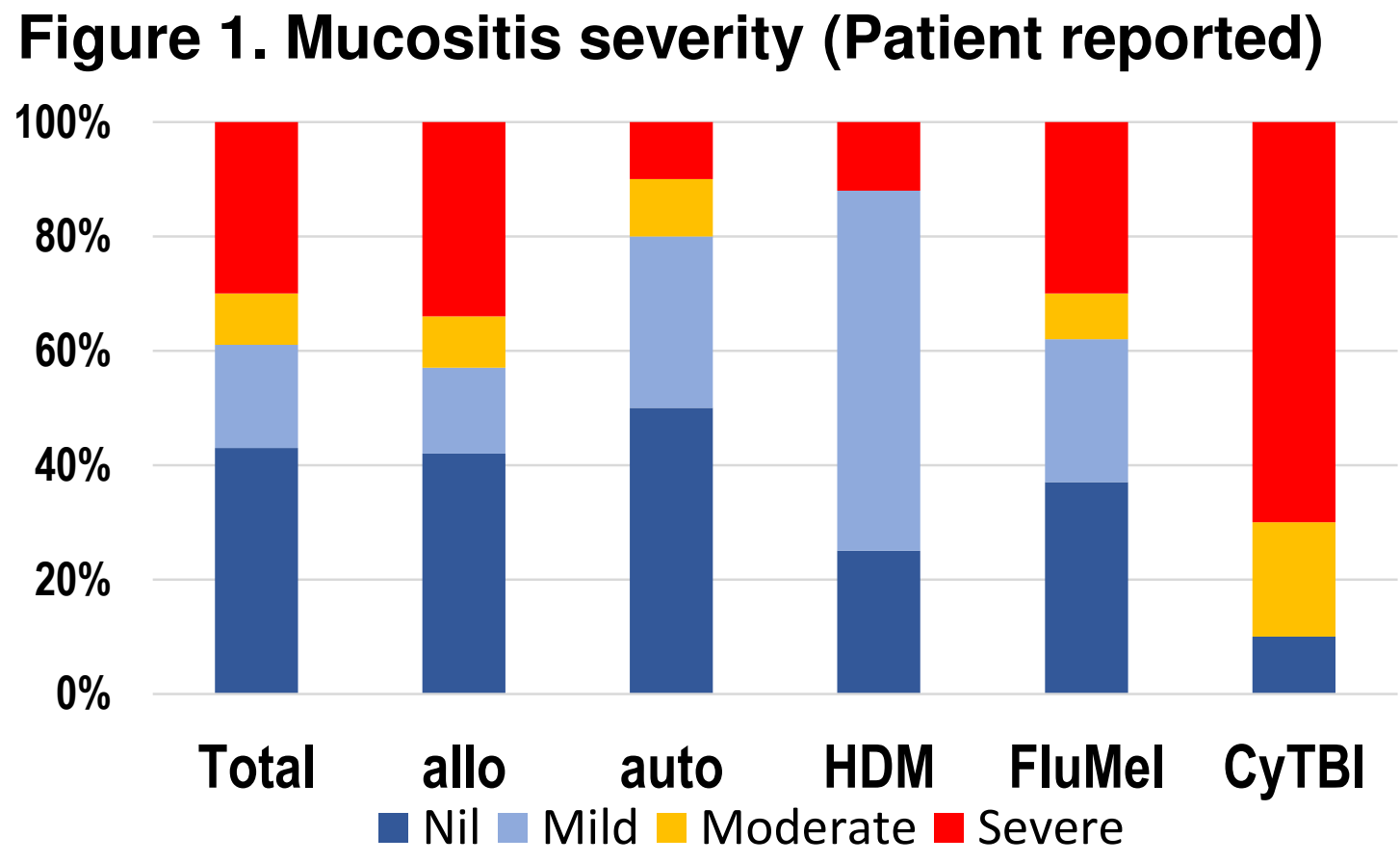

Figure 2. Mucositis severity (WHO grade)

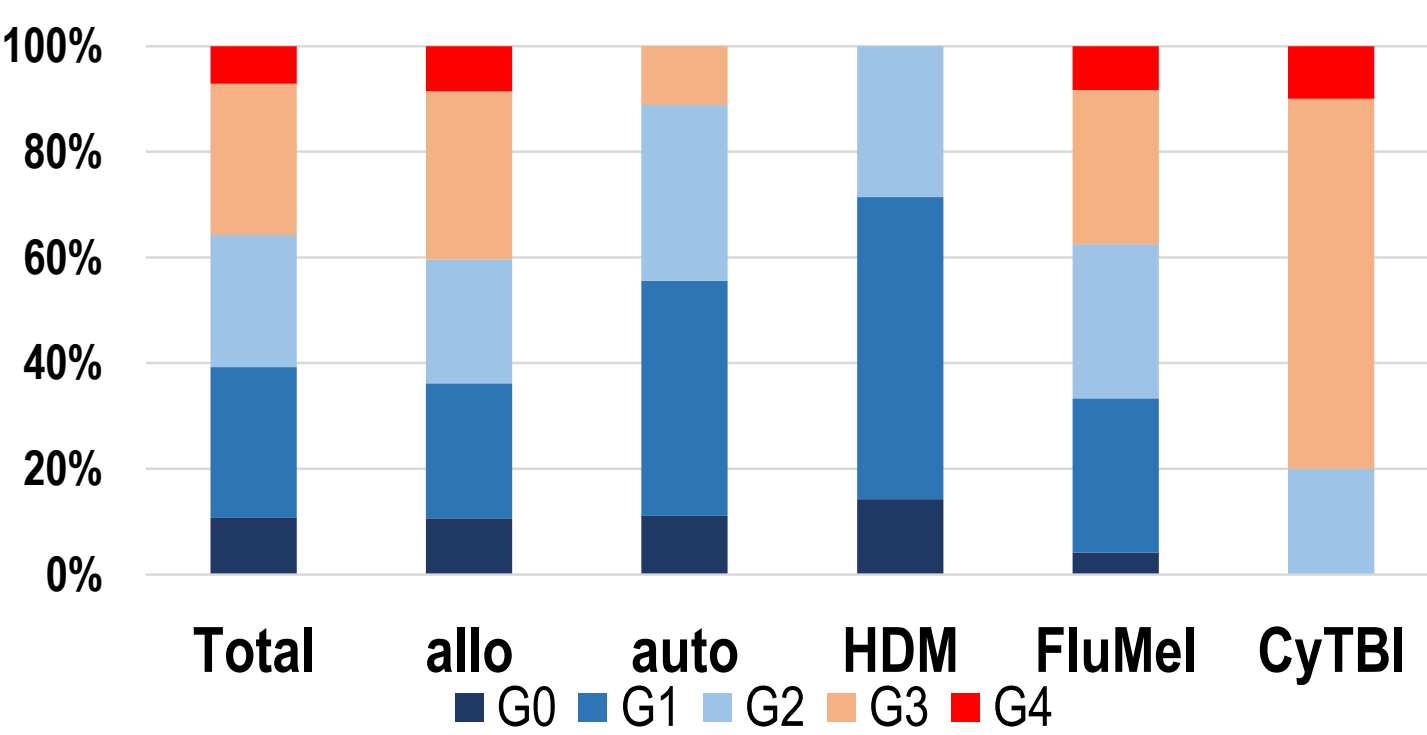

Approximately $40 \%$ had moderate-severe / G3-4 OM. The incidence were allo>auto, HDM>FluMel>CyTBI.

Table 2 . Compliance for cryotherapy

\begin{tabular}{l|l|l|l|}
\hline Protocol & Patients & Cryotherapy & $(\%)$ \\
\hline Melphalan containing & $\mathrm{N}=40$ & 39 & 97.5 \\
\hline Non melphalan containing & $\mathrm{N}=17$ & 5 & 29.4 \\
\hline Total & $\mathrm{N}=57$ & 44 & 77.2
\end{tabular}

Figure 3. Compliance for mouthwashes

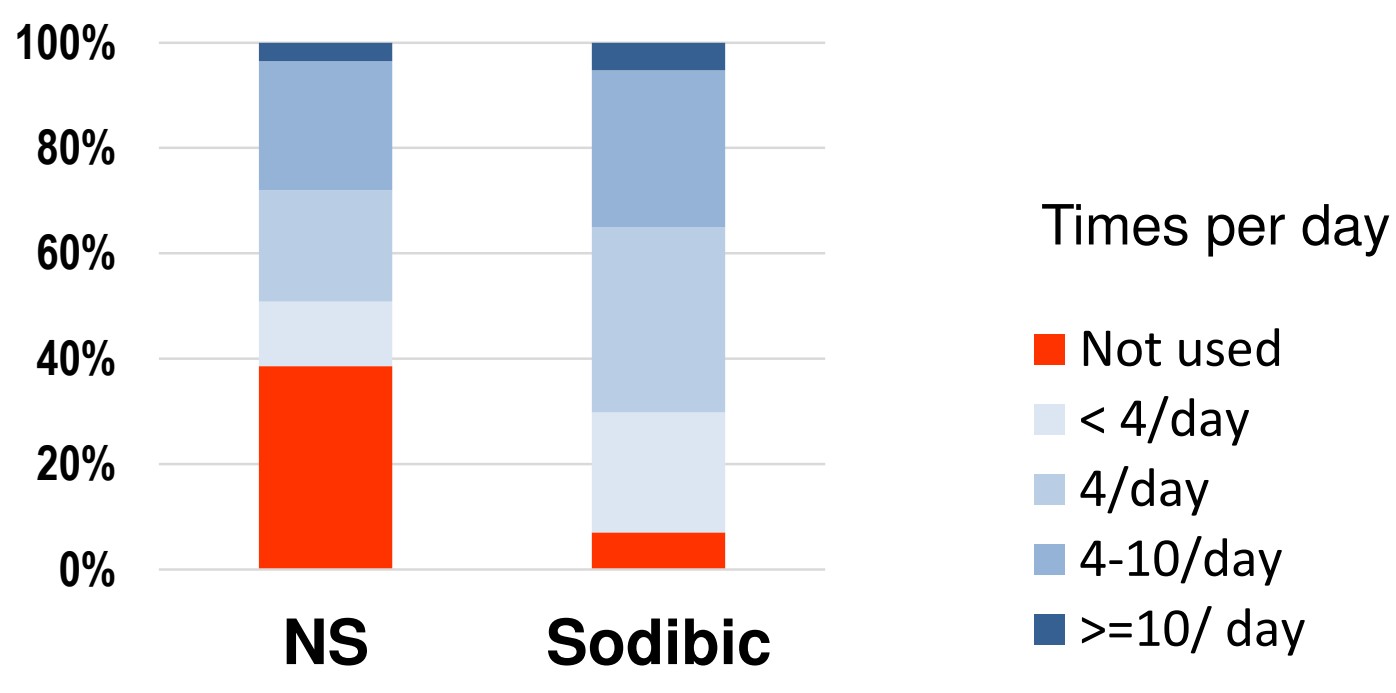

The compliance for cryotherapy and mouthwashes were high (>90\% followed the guidelines). 